\title{
A review on the role of genomics, various environmental factors, biochemical and physiological determinants and biofilms responsible for the development of antibiotics resistance in microbes
}

\author{
Laiba Asif and Sumaira Mazhar \\ Department of Biology, Lahore Garrison University, Lahore-Pakistan \\ *Corresponding author's email: smz.mmg@gmail.com
}

Citation

Laiba Asif and Sumaira Mazhar. A review on the role of genomics, various environmental factors, biochemical and physiological determinants and biofilms responsible for the development of antibiotics resistance in microbes. Pure and Applied Biology. Vol. 9, Issue 4, pp2305-2317. http://dx.doi.org/10.19045/bspab.2020.90245

\begin{tabular}{llll}
\hline \hline Received: 16/03/2020 & Revised: 14/06/2020 & Accepted: 24/06/2020 & Online First: 08/07/2020 \\
\hline \hline
\end{tabular}

\section{Abstract}

Antibiotic (antimicrobial drug), an organic compound causes a sterling impact on microbes by inducing cellular responses. For years, genetic changes were thought to be the only active influencer in development of microbial resistance against antibiotics. With advancement many other factors came up to mutually participate in onset of microbial resistance. This review article is focused on identifying various other possible microbial antibiotic resistance factors. Since there has been identified multitude of factors, the crucial being the horizontal gene transfer (HGT), genetic changes (point mutations), development of biofilms acting as barrier, physiological factors i.e. efflux pump; most effective impact factor, environmental factors; natural selection pressure, metal contamination, anthropogenic activities cooperating specifically with farms serve as vectors to transfer pathogens or their resistance genes to human directly and indirectly through livestock, dairy products consumption, and biochemical responses in terms of mortality and morbidity, working in correlation as a stimulator to each other. To develop a strong antibiotic, it necessitates the knowledge of the evolutionary backgrounds of the microbe. This would help in developing next generation drugs way before the outbreak of next epidemic or pandemic. This requires the development of a gene bank of total resistant genes of pathogens of concern known as resistome. Beneficial bioinformatics databases are in progress to provide useful data in this regard e.g. Antibiotic Resistance Genes Database (ARDB) gathers extensive publicly available info on microbial resistance. Comprehensive Antibiotic Research Database (CARD) generates molecular sequencing data, Antibiotic Resistance Ontology (ARO) and rapidly identifies antibiotic resistance genes in genome.

Keywords: Antibiotic resistance; Antimicrobial Drugs; Biofilms; Cryptic genes; Efflux pumps; Horizontal gene transfer (HGT); Integrating conjugative elements (ICE); Natural selection; Plasmid acquisition MDR efflux pump; resistome.

\section{Introduction}

During the timeline of 1940 to 1960 (golden era of antibiotics), soil microbes Actinomycetes had a huge role in the development of various antibioticsantimicrobial drugs [1]. Soil microbes i.e. Bacillus and Streptomyces were of tremendous scientific research concern due to 
their incredible phenotypic evolution to counter the onset of microbial genetic changes for future antibiotics development beforehand [2]. To combat such rapid evolution in microbes praiseworthy antibiotics with peptide configuration variations, especially the cationic peptide configurations were developed that are hard for microbes to get resistant from [3]. Japan discovered the first transmissible antibiotic resistance gene in microbes holding point mutations responsible for the induction of the antibiotic resistance through plasmid acquisition process [4]. E. coli and S. aureus developed resistance against streptomycin and penicillin and this resistance effect was concluded as the result of point mutation in their genetic makeup [5]. Cryptic genes are such examples of antibiotic resistant resistome in microbes [6] other such example is erm genes [7]. Some antibiotics target the origin of replication in a process called competence, a process efficient in microbes to develop microbial antibiotic resistance as in strain of $S$. pneumoniae. Due to competence about 1 million children die every year of pneumonia [8]. It was found the rates of microbial antibiotic resistance are associated directly to the amount of antibiotic use or exposure and proportional opposite to the health of bacteria [9]. Another experiment was conducted to observe the transfer of resistance among microbes. Bacterial gene (neo genes) that induces resistance to antibiotics; Neomycin and Kanamycin was inoculated into SV40 vectors and then was impregnated into mammalian cells by process called DNA transfusion. Eventually, mammalian cells developed resistance against the antibiotics [10]. The furthermore verification led to the Integrating conjugative elements (ICES); a collection of several mobile conjugative elements from Vibrio cholera, SXT; 100-kilobase (approximately) found to encode genes that helped them to develop resistance not only against multiple antimicrobial drugs; chloramphenicol, sulphamethoxazole, trimethoprim and streptomycin but also against heavy metal resistance genes [11]. The discoveries unveiled that genetic resistance can be innate or acquired. Pseudomonas aeruginosa, depicts a high intrinsic resistance to variety of antibiotic drugs but other microbes are naturally highly antibiotic susceptible, such as group A. streptococci [12]. Hence to collect, assemble and organize this vast information of resistome is a difficult task therefore, bioinformatics databases were developed to store data like Antibiotic Resistance Genes Database (ARDB) that gathers publically available information regarding resistance genome. The database has immense information about resistome, action mechanism, ontology, COG and CDD annotations also available there are external sources about sequence and protein databases [13]. Comprehensive Antibiotic Research Database (CARD) is another bioinformatics database that provides various tools that deal with antibiotic resistance cases in developed in health-care, agriculture, and environmental sectors [14].

Role of environmental factors also resonates with antibiotic resistance development. Natural selection (environmental effecter) is a possible factor that favors the strains with less susceptibility toward the damage by developing resistance through enzymatic inactivation, modification of the antibacterial targets or efflux pumps activations or modifications in microbes [15]. It happened when excess dumping of the disposed antibiotics done openly in the environment which results in the open exposure of antibiotics to microbes, which ultimately act as stimulator for promoting the development and evolution of antibiotic resistance gene. For an effective and vast antibiotic effect, it is recommended to control the usage volume of antibiotic in human health care services and agricultural sectors [16]. Due to 
exaggerated exposure to antibiotics, soil bacteria were very useful in identifying natural and synthetic drug resistance strategies that help antibiotic resistance determinants to evolve and transfer into the microbial community [17]. This was tested and verified in lab when, turbomycin $\mathrm{A}$ and turbomycin B antibiotics were tested on three colonies P57G4, P89C8, and P214D2 of soil bacteria $E$. coli producing that developed resistance against trial cations [18]. It was found that the initiation of development of resistance genes is the response to the scarce concentrations exposures of the antibiotics initially present in the microbial ecosystem provided the initiation signals for increased metabolic responses, especially the extended clinical practices (veterinarian and medical), the high concentration disposed into the environment caused a shift in functional duty. Shifting in the natural environmental laid the foundation for antibiotic resistance genes evolution. For instance, AIDS's opportunistic pathogens would take benefit in such opportunistic environment and attack as they have great resistance for antibiotics. All the species of Pseudomonas aeruginosa containing MDR efflux pump along with ampC beta-lactamase genes exhibits this kind of resistance [16]. Animal husbandries alongside hospitals are also vital in the development of antibiotic resistance in microbes [19]. All the food borne, opportunistic and commensal form of bacterial antibiotic resistance (almost 50\%) attributed to the severe and poor veterinary management conditions [20]. For verification of this fact, farm animals and human were introduced with the transmissible $R$ plasmid of $65 \mathrm{~kb}$ (pTMS1) of coliform bacteria, same plasmid transfused easily in both farm animal and human. The reason stated, due to selective antibiotic pressure in the environment of the farm, multi-drug resistant bacteria was developed that eased the transmission by developing resistance through horizontal gene transfer [21]. Hence, the evolution of resistome in the environment, is the consequence of poor management of disposed of antibiotics especially where animal and human waste is dumped in unaided and unattended in the environment plays a vital role alongside other pollutants that work with mobile genetic elements (resistance elements). Yet, less attention has paid to control such horrific anthropogenic activity [22].

Genetic and environmental factors manipulate the microbes at biochemical and physiological levels. Many biochemical and physiological effectors are correlating responsible for the development of antibiotic resistance. The most important knowledge so far has obtained in this regard is in terms of mortality and morbidity [15]. For the antibiotic biochemical and physiological resistance mechanism, a number of three strategies are used by antibiotic producing microbial strains; demolishing the antibiotic, efflux pump action and the structural modification of the antibiotic targeted cellular entities [23]. The target of the most antibiotics is the outer membrane. Antibiotics work in two metabolic pathways to get through the outer plasma membrane: 1 . for hydrophobic antibiotics; a lipid-mediated pathway, and 2. for hydrophilic antibiotics; diffusion porins [24]. Multidrug efflux systems help in this regard as they are broad spectrum efflux systems hosting resistance to structurally differentiated broad variety of antimicrobial antibiotics. These pumps in inner plasma membrane contain an antiporter that's a drug proton antiporter and in outer plasma membrane with periplasm spanning channel forming protein along a periplasmic associated protein that joins them together [25]. In bacteria that carry the resistance gene acr $A B$ on their plasmid, over expression of the acr $A B$ was responsible for acquiring resistance. The efflux pump makes these bacteria easily susceptible to antibiotics. 
Hence, to combat that, CmeABC efflux resistance pump is present in Campylobacter jejuni, PmrA in Streptococcus pneumonia, NorA in $S$. aureus. Mutation in the repressor genes was deemed as the cause of the over expression for the efflux pump genes or the stimulation of the regulon by global transcriptional regulator, for instance, the MarA and SoxS in E. coli [26]. Antibiotic resistance gene alongside the resistance determinant is efficient for gaining ecological benefits to the microbe for colonizing in its environment. The resistant determinants induce selection pressure either by pollution caused by heavy metal or chemicals. Much of the determinants are manufactured naturally in the form antibiotic resistance genes by organisms that are antibiotic producers themselves. In Mycobacteria, there are otrA and $\operatorname{otr} B$ determinants for this purpose [27]. The antibiotic resistance may result in interbacterial antagonism which is a consequence of the virulence, production, phage induction, SOS stress response, cell to cell signaling, self-growth regulation, mutagenesis, intrachromosomal transduction, mortality, sporulation, motility, lateral gene transformation, and biofilm formation [28]. Biofilms are self-formed by the association of the microbial populations sticking together by exopolysaccharides (EPS). Biofilm is a polymer-matrix made up of polysaccharide, protein with DNA. It causes chronic infections with increased tolerance to supplied antibiotics and disinfectants resisting to phagocytosis [29]. These biofilms have up to a 1000-fold greater antibiotic resistance than planktonic cells. But it was found that $P$. aeruginosa doesn't develop high-level biofilm resistance as $n d v B$ is required for the production of periplasmic glucans polymers essential to forbid antibiotics from penetrating their action sites in the periplasm [30]. For instance, in Cystic fibrosis, a chronic lung infection occurs because of Pseudomonas aeruginosa biofilm-growing mucoid strains. Biofilm increased formation is the result of mutations and quorum-sensing-regulation. Other resistance mechanisms such as upregulated efflux pumps, chromosomal $\beta$-lactamase also participate to the survival of biofilms for antibiotics resistance [29]. Bacteria that grow as a biofilm are secured from antibiotics. Improper antibiotic penetration, limited nutrient, slow growth, stress response adaptation, and constitution of persister cells are responsible for biofilm defense mechanism [31]. There have been many factors co-working to bring about the microbial antibiotic resistance. Genomics, environmental factors, several different determinants and biofilms work in collaboration to persist this phenomenon.

\section{Genomics role in antibiotics resistance}

1940 to 1960 is regarded as the Golden era of antibiotics. Actinomycetes were first examined from soil for the extraction of most of the antibiotics [1] as soil microbes show noteworthy phenotypic evolution it was best to study for future aims [2]. The first transmissible antibiotic resistance gene was reported in Japan and resistance was attributed to the point mutations in genomic sequence [4]. Antibiotic exposure imposes a decline in growth rate of microbes reciprocal to rates of exposure [9]. Cryptic genes [6], erm genes [7], neo genes [10], integrating conjugative elements (ICE) [11] etc exhibit a vast variety of resistome and microbes use several strategies to induce genetic resistance against antibiotics such as horizontal gene transfer (HGT), competence, and via DNA transfusion etc.

Role of genome, genomic units and different genomic strategies

Bacterial antibiotic resistance to the streptomycin and penicillin was checked on NRRL 313 strain of S. aureus and E. coli suggested the genetic mutations being the cause of resistance. The development of resistance was attributed to the exposure time 
of contact between antibiotics and microbes that triggered the genetic mutations. Different bacterial strains developed different level of resistance in response to it, as the strain that was resistant to streptomycin was not so resistant to penicillin. This triggered the genetic mutations in the bacterial strains acquiring resistance or immunity [5] and discovered that the development of the antibiotic microbial resistance depends upon the amount of antibiotic exposure to the microbes and not to the microbial health cost [9]. To understand this, first transmissible resistance gene was identified in Japan. Plasmid acquisition's coassociation with genetic variations induced by point mutation was recognized for incorporating microbial genetic modification for adapting resistance [4]. The development and evolution of the resistance genes are correlated by Cytotoxic antimicrobial agents involved in inducing certain biochemical activities that triggered the production of a precursor protein as a powerful selection pressure. These biochemical activities were responsible for the evolution of the main microbial resistance genes that co-works with other resistance cryptic genes in resistome [6]. This was supported when various genetics strategies such as Chromosomal changes, genetic material exchange thorough plasmids and transposons in Streptococcus pneumoniae, Streptococcus pyogenes, and Staphylococci and Enterobacteriaceae and Pseudomonas families were found to bring resistance among them [32]. Other biochemical and physiological strategies were identified as methylation of $23 \mathrm{~S}$ rRNA, lactone ring hydrolysis, removal of efflux pump, point mutation for inducing resistance in enterococci, staphylococci, streptococci, as the result of the horizontal gene transfer (HGT) introducing erm resistance genes family [7]. Nearly 1 million children die every year of pneumonia due to $S$. pneumoniae has developed resistance in response to competence, a process of targeting the origin of replication (oriC) located near the ori of DNA replication that led to the activation of the genes of resistance [8].

Mobile genetic elements (integrating conjugative elements (ICEs) and multiple artificial systems to identify resistome in microbes

For the study of antibiotic resistance phenomenon in microbes many artificial systems are designed that would help in understanding naturally acquired resistance in microbes. For the study of the resistome, four broad host range (bhr) cloning plasmid vectors $p B B R 1 M C S$ were artificially introduced that were so tiny $(<5.3 \mathrm{~kb})$, with multiple cloning site (MCS), compatible with ColE1, P15a replicons and IncP, IncQ, IncW plasmids, allow direct selection of plasmid through interference of $\mathrm{LacZ} \alpha$ peptide in $E$. coli [33]. Other strategic elements for the study of resistance involve mobile genetic elements or integrating conjugative elements (ICEs) regulate conjugative transfer of resistome and induce SOS response to DNA damage by SetR that's a SXT repressor, represses activators of SXT transfer. SXT is a 100-kilobase ICE derivate discovered in 1993 from Vibrio cholerae encodes resistance genes for sulphamethoxazole, chloramphenicol, streptomycin, and trimethoprim [11]. This led to PCR assays to identify nine more resistance genes on thirty different isolates of Staphylococcus aureus namely mecA (encoding methicillin resistance), aacA-aphD (aminoglycoside resistance), tetK, tetM (tetracycline resistance), erm(A), erm(C) (macrolidelincosamide streptogramin $B$ resistance), $\operatorname{vat}(\mathrm{A}), \operatorname{vat}(\mathrm{B})$, and $\operatorname{vat}(\mathrm{C})$ (streptogramin $\mathrm{A}$ resistance) [34]. In Salmonella typhimurium phage DT104, resistance genes involve integrons. ACSSuT resistant phenotype genes have been cloned for detection of multiresistant DT104 phage [35]. Two 
cloning plasmids pUC18Not and pUC18Sfi were manufactured by combining two plasmids inoculated into gram-negative eubacteria resulting NotI or SfiI sites used for DNA cloning. In this cloning system a kanamycin resistance gene was inserted into Pseudomonas putida with a gene coding for melanin from Streptomyces antibioticus and Klebsiella pneumoniae [36].

\section{Environmental factors}

Soil bacteria are very useful in studying various microbial strategies occurring in natural environment that determine the determinants causing evolution and transfer of the antibiotic resistance via phenotypic variations [2]. Improper waste management due to disguised anthropogenic activities of veterinarians, medical practitioners, and dairy farm farmers has contributed a lot in microbial resistance havoc. Not less important but the anthropogenic impacts induced by large industries, automobiles and metal/chemical contaminations are huge environmental influencer to the microbial communities as well [22].

\section{Natural selection pressure}

The rule of natural selection (environmental effector) is the survival of the fittest. Natural selection pressure is identified to favor the strains of microbes with less susceptibility to antibiotics by developing enzymatic inactivations, modifications of the antibacterial targets or efflux pumps [15]. Due to impact of natural selection the survival of the fittest microbes has been favored on fitness cost. This fitness cost is evaluated by peculiar resistance mutations and genetic profile. In Mycobacterium tuberculosis, resistance against Rifampin is the perfect example of the competitive fitness cost phenomenon [37].

\section{Metal and chemical contaminations}

Metal and chemical contaminations $(\mathrm{Cu}, \mathrm{Ar}$, CFCs etc.) play a role as a selective agent which determines the mechanism to transfer resistance in microbes. This is a co-selection procedure including co-resistance; the existence of different resistance determinants on same genetic element and cross-resistance that is the same genetic determinants responsible for resistance, to antibiotics and metals [38]. Major amount of antibiotics has been introduced from human derived activities. Any change into the natural environment could lead to the disturbance in the population dynamics that would cause unpredicted consequences by pathogens and antimicrobial drug resistance in microbes. This disturbance would lead to advanced antibiotically-evolved microbial communities and havoc for the drugs and procedures for treatments of the ailments would occur leading to the necessity of new generation drugs with unknown strategies acquired and developed in microbes [39].

Anthropogenic activities, farm and aqua industries playing as environmental antibiotic resistance vectors, antibiotic resistance genes communication by conjugation

A study conducted on river sediments at Cache La Poudre River, Colorado, United States, a correlating relationship was found between urban activities, agricultural activities. Antibiotics tetracyclines and sulfonamides were found disposed of in the river unattended provoking development of resistance in the naturally existing microbes at the sites. Microbial populations were effected according to the volumes of antibiotic exposures at different sites of the river [40]. These antibiotics were used for over growth of animals and control of infections at animal husbandries. Poorly handled animal waste was suggested as the biggest hazard among others for the outbreak of antibiotic resistance promotion [41]. Farm ecosystems are natural hub for resistome development, especially the poorly managed ones acting as the ultimate vector for transferring potentially harmful resistome to humans and other living beings through 
consumption of dairy products and meat or when animal's dead carcasses left poorly or openly disposed of in the environment. It's also being a cause of de novo mutations in microbes. This phenomenon works well with Mutual Corporation of mobile genetic elements (resistance elements/ICEs), environmental pollutants, natural reservoir resistome and genetic mutations to induce a havoc of microbial resistance [22].

In a PCR study, results suggested dairy cheese and sheep feces were contaminated with esp surface gene, AS gene, colonization factor ace, endocarditis antigen efaA, gelatinase gelE gene were accounted for in the virulence of the Enterococcus faecium making them pathogenic [42]. Hence, $50 \%$ of all the food borne, opportunistic and commensal form of bacterial antibiotic resistance was awarded to mismanaged veterinary outcomes [20]. To get effective antibiotic effect, limited usage of antibiotics for human medicinal purposes and in agricultural domains is necessary as the excess use leads to evolution and development of resistance genes in microbes in environment through exposure [16]. Half of the antibiotic resistance in ecosystem is due to the improper handling of vaccine antibiotic treatments given to farm animals. Several incidences have occured to put an eye on such as, antibiotic-resistant enterococci and staphylococci from animals could survive the food-making processes such as in raw-cured sausages and raw-milk cheeses. The broad host ranges (bhr) plasmids and transposons in many bacteria allow antibiotic-resistance genes to communicate by conjugation between different species and genera. A multi-antibiotic resistance plasmid from a lactococcus found in cheese provides a historical record of such events [43]. Poor hospital conditions i.e. Intensive care units (ICU) at health sectors are also a hub to a lot of antibiotic resistant microbes as a result of excessive and unnecessary usage of antimicrobial agents, poorly disposed of waste and uncured medical stations [44]. This gives rise to concept of clinical resistance in microbes [45].

Moreover, not only land but waters are not safe to such diversifying microbial changes. Large fish business is built on fish industries, aquaculture industries are also identified as carrying the microbial infections that were subjected to vaccines to immune fish from various diseases but later on it was found the natural and parasitic microbes on fish developed the antibiotic resistance in return [46] proving the aquatic environments are also ideal for emergence of novel antibiotic resistance genes (ARGs) done so through horizontal gene exchange (HGT) for mobile genetic elements (ICEs) [47].

\section{Biochemical and physiological determinants}

Several other determinants have been found mainly dealing with physiology and biochemistry of microbes. In order to infect any microbes by antibiotics, it's compulsory to disturb its physiology and biochemistry by inducing structural modifications of the antibiotic targeted cellular entities and destroying microbe's efflux pumps most importantly but microbes counter attack to retain them [23].

\section{Outer membrane systems to adapt antibiotic resistance}

Microbes use three different mechanisms to counter attack antibiotics 1: destroying the antibiotics being administered, 2: the action of its efflux pump and 3: the structural modification of cellular bodies targeted by antibiotic [23]. For any antibiotic to penetrate and disturb the internal machinery hub of a microbe it's important to penetrate its outer membrane systems that are favorite site for antibiotics to attack in microbes it happens so by attacking the lipid stimulated pathways and by common diffusion Antibiotics get through the outer plasma membrane by two ways: 1. for hydrophobic antibiotics; a lipid- 
mediated pathway is used, and 2. for hydrophilic antibiotics; diffusion porins are used [24].

\section{Efflux pump systems}

Zoonotic ailments play a magnificent role in triggering the rise of antibiotic resistant microbes (bacteria) [48]. In bacteria, the genes for antibiotic resistance are found on their plasmids. In such bacteria, efflux pumps are crucial as they are susceptible to the antibiotics detection for stimulating resistance such as $C m e A B C$ in Campylobacter jejuni, PmrA in Streptococcus pneumonia, NorA in S.aureus are efflux pumps. Efflux pumps got their antibiotic resistant role due to the local mutation and failed control of the repressor genes that led the over expression of the genes encoded for efflux pumps, few examples in E. coli efflux pumps are the MarA and SoxS [26]. Efflux pumps could be multiude in function such that in E. coli $K$ 12; isogeneic strains that are multiple antibiotic resistance (Mar) mutants acquiring resistance to multiple drugs and have multi efflux pumps to deal multi-drug situation. These synthetic mutants were manufactured by AcrAB system pump as major contributor. Hence, E. coli strains are immune to tetracycline, chloramphenicol, ampicillin, nalidixic acid, and rifampin antibiotic drugs [49]. There also exists a multidrug efflux system (MES) that can target even unrelated species and provide resistance to many possible antibiotics. Such systems provide efficient response not only for antibiotics but also for biocides such that triclosan. For example, opportunistic pathogen Pseudomonas aeruginosa provides both intrinsic and acquired resistance to triclosan [25].

\section{Role of chemotherapy in natural and acquired resistance}

There is a role of chemotherapy in the evolution of the microbes to acquire natural antibiotic immunity. The evolutionary units such as genes, integrons, transposons, plasmids, cells, communities, microbiomes, hosts played a vital role in the evolution of resistance among microbes. To survive in the environment these units evolved that in return benefited the microbes with development of antibiotics resistance machinery systems. Bacteria also produce themselves antibiotics naturally to sustain in counter to environmental stresses and contamination. As microbes live in colonies this provided the chance to inherit resistance to other microbes as well. Biofilm production, SOS response, mortility, cellular signaling, self-growth regulation, mutagenesis, virulence, phage induction, intra-chromosomal transduction, sporulation, motility, lateral gene transformation are the factors to induce interbacterial antagonism [28]. Since the start of antibiotic era, the microbes were susceptible to adapt acquired antibiotic immunity mostly in the bacteria that were inherently susceptible to antibiotics, and phenotypically responded to such stimulus but there is also non-inherited microbial antibiotic resistance that could trigger patient's treatment failure response [50].

\section{Horizontal gene transfer (HGT)}

Horizontal gene transfer (HGT) is the inheritance of genetic material among microbial communities also known as lateral gene transfer is a giant contributor in grasping the understanding of evolution, development and inheritance of antibiotics resistance [51]. It's crucial in identifying resistance mechanisms in virulent microbial communities. Since antibiotics target the genes in plasmid; a circular genetic entity, HGT was very useful in the investigation. It was revealed by HGT many other determinants were also responsible for this alteration in the microbes acquiring antibiotic resistance, such as heavy metals and chemical pollution. Antibiotics resistance genes were responsible for giving microbial 
communities ecological benefits, for instance, bearing the selection pressure, defense mechanisms, retaining populations by colonization etc. Microbes naturally produce these determinants themselves also such that otrA and otrB for tetracycline resistance in Mycobacteria [27].

Role of DNA-complex Tet-repressor, Ethidium bromide and Acridine in eliminating antibiotic resistance

As efflux pumps are vital role players in resistance hence modifying the pumps could help in inverting the evolved physiological determinant in microbes. The distortion of the DNA-complex Tet-repressor encourages the expression of the Tet A resistance protein. This activates the efflux pumps resisting tetracycline in gram negative bacteria. The crystal structure of Tet repressor combined with tetracycline-magnesium made 2.5 angstrom entity. The helix-turn-helix motifs of the Tet repressor involved in operatorbinding were inverted in contrast to any other DNA binding proteins. The repressor-drug complex couldn't act together with DNA due to 5 angstroms partition of the DNA binding motifs hence can't inherit or express [52]. Ethidium bromide was recognized for eliminating some antibiotics resistance in microbes. Penicillinase manufacturing was eradicated in staphylococci mercury-resistant organisms. In E. coli KI2 strains, the $F^{\prime}$-lac+ factor was eradicated by using ethidium bromide. Acridine dyes also gave high potential results in eradicating antibiotic resistance [53].

\section{Biofilms}

Biofilms are sticky to the surfaces due to extracellular materials in composition with DNA and protein [29]. It's a complex structure formed by mutual association of microbial community [54] helped them to tolerate antibiotics [55]. Biofilms have 1000 times' greater efficiency against antibiotics than a planktonic cells. A very important constituent of biofilm production is $n d v B$ for the production of EPS that prevents penetration of antibiotics in their action sites in the periplasm [30].

Resistance associated with mutations, quorum-sensing-regulated mechanisms, and role of periplasmic glucans (EPS) and MDR efflux pumps:

Biofilm growth is synced with greater mutations and quorum-sensing-regulated mechanisms. It is responsible for chronic infections and high tolerance to antibiotics. They resist host's defensive phagocytosis. Staphylococcal infections persist due to their biofilm formation. In cystic fibrosis, Pseudomonas aeruginosa causes chronic lung infection by developing biofilmgrowing mucoid strains. As biofilms have efficient EPS periplasm they resist any change to them. In addition to EPS, cellular aggregates with less or more dormancy are accountable for antibiotic resistance. The survival of biofilms depends upon chromosomal $\beta$-lactamase, upregulated efflux pump mutations. To prevent biofilm production following strategies are worthy; prophylaxis, chronic suppressive therapy, use of DNase and alginate lyase to dissolve the biofilm polymer-matrix and quorumsensing inhibitors [29]. Pseudomonas aeruginosa biofilms were examined lacking the MexAB-OprM MDR efflux pump. Multidrug antibiotic resistance efflux pumps (MDR)'s absence was found crucial in the biofilm resistance meachnism. For instance, ofloxacin resistance was relying on the MDR expression of MexAB-OprM however ciprofloxacin resistance did not depend on MDR in biofilms [56]. Biofilms also get their benefit of multilayer protection from antibiotic's inadequate penetration, limited nutrient and dim growth rate, stress responses adaptively, and persister cells development [31]. Periplasmic glucans (EPS) majorly correlate to maintain a physical interaction with antibiotics such that to tobramycin and prevent it from diffusing into the biofilm. 
This suggested that glucose polymers (EPS) were responsible for preventing antibiotics from diffusing action [30].

Role of biofilm's physiology and biochemistry to cationic antimicrobial peptides

In biofilms, DNA expression is given the correct physiological conditions to initiate antimicrobial properties including cell lysis to stabilize lipopolysaccharide (LPS) and outer membrane. DNA induces disturbances to inner and outer membrane systems, hence, have minutes' response rate accompanied with release of cytoplasmic nucleic acid; DNA. This creates a cation scarce environment. In $P$. aeruginosa, Cationic peptide resistance operon PA3552 PA3559 are regulated by $P h o P Q$ and $P m r A B$. This resulted in up to 2560 times higher cationic antimicrobial peptides resistance and 640 times higher aminoglycosides resistance [57].

\section{Conclusion and recommendations}

Antibiotic resistance is accomplished in microbes by multiple factors that cooperate with each other to initiate different resistance mechanisms. All these factors are mutually dependent and correlated to trigger resistance in microbes. Horizontal gene transfer, competence, plasmid acquisitions, point mutations, natural selection pressure, metal and chemical contaminations, anthropogenic activities such poor and improper disposal of waste and antibiotic products both by hospitals and animal husbandries, efflux pumps, MDR pumps, biofilm periplasmic glucans (EPS) and cell lysis are the main stimulators to induce antibiotic resistance is microbes. Microbes developed resistance initially naturally in response to the environmental pressure factors to survive i.e. natural selection, evolution, race to colonization. For this regard, they inherited the resistance to next generations by genetic units. Later on, anthropogenic activities stimulated the greater resistance havoc.
Animal husbandries' poor conditions, unattended antibiotics' disposal in the environment constitutes $50 \%$ of the resistance stimulation. The strength of the resistance depends upon the amount and time of exposure of the antibiotics to microbial community ecosystems hence stimulating the genetic units for adapting resistance genes (resistome) that are in fact the microbial defense responses.

The understood cycle for the development of resistance sought out from the review is as the environmental selection pressure for survival served the first stimulator of the genetic changes in the microbes alongside point mutations in the microbial genome played a crucial role. These genetic changes induced several other changes in physiology and biochemistry of microbes generating and modifying efflux pump systems, biofilm periplasm EPS, various resistome genes i.e. erm genes, cryptic genes, ICEs etc. each stimulator introduced a new mechanism to inherit the change for survival (antibiotic resistance) namely horizontal gene transfer, competence, plasmid acquisitions, point mutations, natural selection pressure, metal and chemical contaminations, anthropogenic activities such poor and improper disposal of waste and antibiotic products both by hospitals and animal husbandries, efflux pumps, MDR pumps, biofilm periplasmic glucans (EPS) and cell lysis. The increased anthropogenic activities and needs further pushed this evolutionary genetic physiological and biochemistry miracle to happen. The best example of it is Biofilms that cause 1000 folds more effect than planktons. Not a single factor works in isolation rather needs every factor to work in collaboration to bring about such a dramatic antibiotic resistance phenomenon.

To eliminate the resistance in microbes Ethidium bromide and Arcdine have been successful to some extent so far. Acridine dyes gave high potential results in wiping off 
antibiotic resistance. Penicillinase enzyme was eradicated in staphylococci mercuryresistant organisms also in E. coli KI2 strains, the $F^{\prime}$-lac+ factor was eliminated using Ethidium bromide. Another suggested way is to change the efflux pump structures. The 2.5 angstrom crystal structure of Tet repressor with tetracycline-magnesium is normal efflux pump. The operator-binding helixturn-helix motifs of the Tet repressor were changed to 5 angstroms. The repressor-drug complex couldn't interact with DNA due to 5 angstroms size of the altered DNA binding motifs. Reduced environmental pollution and proper discharge of the antibiotics from both medical centers of humans and animal husbandries should be checked and balanced. For this purpose, laws must be made and implemented. Dead carcasses of the animals must be buried properly and not left untreated in open environments, left over used vaccines and medical wastes must be incriminated properly.

\section{Authors' contributions}

Conceived the idea: Laiba Asif and Sumaira Mazhar, Wrote the paper: Laiba Asif and Sumaira Mazhar, Corresponding author: Sumaira Mazhar

\section{References}

1. Lewis K (2013). Platforms for antibiotic discovery. Nat Rev Drug Disc 12(5): 371.

2. Traxler MF \& Kolter R (2015). Natural products in soil microbe interactions and evolution. Nat Prod Rep 32(7): 956-970.

3. Hancock R E (1997). Peptide antibiotics. The Lancet 349(9049): 418-422.

4. Davies JE (1997). Origins, acquisition and dissemination of antibiotic resistance determinants. In Ciba Found Symp 207: 1527.

5. Demerec M. (1948). Origin of bacterial resistance to antibiotics. J Bacteriol 56(1): 63.

6. Wright GD (2007). The antibiotic resistome: the nexus of chemical and genetic diversity. Nat. Rev Microbiol 5(3): 175.
7. Jensen LB, Frimodt-Moller N \& Aarestrup FM (1999). Presence of erm gene classes in gram-positive bacteria of animal and human origin in Denmark. FEMS Microbiol Lett 170(1): 151-158.

8. Slager J, Kjos M, Attaiech L \& Veening JW (2014). Antibiotic-induced replication stress triggers bacterial competence by increasing gene dosage near the origin. Cell 157(2): 395-406.

9. Andersson DI \& Levin BR (1999). The biological cost of antibiotic resistance. Curr Opin Microbiol 2(5): 489-493.

10. Southern PJ \& Berg P (1982). Transformation of mammalian cells to antibiotic resistance with a bacterial gene under control of the SV40 early region promoter. J Mol App Gen 1(4): 327-341.

11. Beaber JW, Hochhut B \& Waldor MK (2004). SOS response promotes horizontal dissemination of antibiotic resistance genes. Nat 427(6969): 72.

12. Normark BH \& Normark S (2002). Evolution and spread of antibiotic resistance. J Intern Med 252(2): 91-106.

13. Liu B \& Pop M (2008). ARDB - antibiotic resistance genes database. Nucleic Acids Res 37(1): D443-D447.

14. McArthur AG, Waglechner N, Nizam F, Yan A, Azad MA, Baylay AJ \& Kalan L (2013). The comprehensive antibiotic resistance database. Antimicrob Agents Chemother 57(7): 3348-3357.

15. Livermore DM (2003). Bacterial resistance: origins, epidemiology, and impact. Clin Infect Dis 36(1): S11-S23.

16. Martinez JL (2009). The role of natural environments in the evolution of resistance traits in pathogenic bacteria. Proc $R$ Soc London B 276(1667): 2521-2530.

17. D'costa VM, McGrann KM, Hughes DW \& Wright GD (2006). Sampling the antibiotic resistome. Sci 311(5759): 374-377.

18. Gillespie DE, Brady SF, Bettermann A D, Cianciotto NP, Liles MR, Rondon MR ... \& Handelsman J (2002). Isolation of antibiotics turbomycin $\mathrm{A}$ and $\mathrm{B}$ from a metagenomic library of soil microbial 
DNA. Appl Environ Microbiol 68(9): 4301-4306.

19. Witte W (1998). Medical consequences of antibiotic use in agriculture. Sci 279(5353): 996-997.

20. Teuber M (2001). Veterinary use and antibiotic resistance. Curr Opin Microbiol 4(5): 493-499.

21. Oppegaard H, Steinum TM \& Wasteson Y (2001). Horizontal transfer of a multi-drug resistance plasmid between coliform bacteria of human and bovine origin in a farm environment. Appl Environ Microbiol 67(8): 3732-3734.

22. Wellington EM, Boxall AB, Cross P, Feil EJ, Gaze WH, Hawkey PM... \& Thomas C $M$ (2013). The role of the natural environment in the emergence of antibiotic resistance in Gram-negative bacteria. Lancet Iinfect Diseas 13(2): 155-165.

23. Walsh C (2003). Antibiotics: actions, origins, resistance. (ASM) 13(11): 30593060.

24. Delcour AH (2009). Outer membrane permeability and antibiotic resistance. $\mathrm{Bba}$ proteins proteom 1794(5): 808-816.

25. Poole K (2002). Mechanisms of bacterial biocide and antibiotic resistance. $J$ Appl Microbiol 92: 55S-64S.

26. Webber MA \& Piddock LJV (2003). The importance of efflux pumps in bacterial antibiotic resistance. $J$ Antimicrob Chemother 51(1): 9-11.

27. Alonso A, Sanchez P \& Martinez JL (2001). Environmental selection of antibiotic resistance genes: Minireview. Environ Microbiol 3(1): 1-9.

28. Baquero F, Tedim ASP \& Coque TM (2013). Antibiotic resistance shaping multi-level population biology of bacteria. Front Microbial 4: 15.

29. Hoiby N, Bjarnsholt T, Givskov M, Molin S \& Ciofu O (2010). Antibiotic resistance of bacterial biofilms. Int $J$ Antimicro Agents 35(4): 322-332.

30. Mah TF, Pitts B, Pellock B, Walker GC, Stewart PS \& O'toole GA (2003). A genetic basis for Pseudomonas aeruginosa biofilm antibiotic resistance. Nat 426(6964): 306.
31. Stewart PS (2002). Mechanisms of antibiotic resistance in bacterial biofilms. Int J Med Microbiol 292(2): 107-113.

32. Neu HC (1992). The crisis in antibiotic resistance. Sci 257(5073): 1064-1073.

33. Kovach ME, Elzer PH, Hill DS, Robertson GT, Farris MA, Roop II R M \& Peterson KM (1995). Four new derivatives of the broad-host-range cloning vector pBBR1MCS, carrying different antibioticresistance cassettes. Gene 166(1): 175-176.

34. Strommenger B, Kettlitz C, Werner G \& Witte W (2003). Multiplex PCR assay for simultaneous detection of nine clinically relevant antibiotic resistance genes in Staphylococcus aureus. J Clin Microbiol 41(9): 4089-4094.

35. Briggs CE \& Fratamico PM (1999). Molecular Characterization of an Antibiotic Resistance Gene Cluster of Salmonella typhimurium DT104. Antimicrob Agents Chemother 43(4): 846849.

36. Herrero M, de Lorenzo V \& Timmis KN (1990). Transposon vectors containing non-antibiotic resistance selection markers for cloning and stable chromosomal insertion of foreign genes in gram-negative bacteria. J Bacteriol 172(11): 6557-6567.

37. Gagneux S, Long CD, Small, PM, Van, T, Schoolnik G K \& Bohannan BJ (2006). The competitive cost of antibiotic resistance in Mycobacterium tuberculosis. Sci 312(5782): 1944-1946.

38. Baker-Austin C, Wright MS, Stepanauskas R \& McArthur JV (2006). Co-selection of antibiotic and metal resistance. Trends Microbiol 14(4): 176-182.

39. Martínez JL (2008). Antibiotics and antibiotic resistance genes in natural environments. Sci 321(5887): 365-367.

40. Pei R, Kim SC, Carlson KH \& Pruden A (2006). Effect of river landscape on the sediment concentrations of antibiotics and corresponding antibiotic resistance genes (ARG). Water Res 40(12): 2427-2435.

41. Economou V \& Gousia P (2015). Agriculture and food animals as a source of 
antimicrobial-resistant bacteria. Infect Drug Resist 8: 49.

42. Mannu L, Paba A, Daga E, Comunian R, Zanetti S, Duprè I \& Sechi LA (2003). Comparison of the incidence of virulence determinants and antibiotic resistance between Enterococcus faecium strains of dairy, animal and clinical origin. Int J Food Microbial 88(2-3): 291-304.

43. Perreten V, Schwarz F, Cresta L, Boeglin M, Dasen G \& Teuber M (1997). Antibiotic resistance spread in food. Nat 389(6653): 801.

44. Kollef MH \& Fraser V J (2001). Antibiotic resistance in the intensive care unit. Ann Int Med 134(4): 298-314.

45. Hawkey PM (1998). The origins and molecular basis of antibiotic resistance. BMJ 317(7159): 657-660.

46. Alderman DJ \& Hastings TS (1998). Antibiotic use in aquaculture: development of antibiotic resistance-potential for consumer health risks. Int J Food Sci Technol 33(2): 139-155.

47. Marti E, Variatza E \& Balcazar JL (2014). The role of aquatic ecosystems as reservoirs of antibiotic resistance. Trends Microbiol 22(1): 36-41.

48. Allen HK, Donato J, Wang HH, CloudHansen KA, Davies J \& Handelsman J (2010). Call of the wild: antibiotic resistance genes in natural environments. Nat Rev Microbial 8(4): 251.

49. Okusu H, Ma D \& Nikaido H (1996). AcrAB efflux pump plays a major role in the antibiotic resistance phenotype of Escherichia coli multiple-antibioticresistance (Mar) mutants. $J$ Bacterial 178(1): 306-308.
50. Levin BR \& Rozen DE (2006). Noninherited antibiotic resistance. Nat Rev Microbiol 4(7): 556.

51. Davies J \& Davies D (2010). Origins and evolution of antibiotic resistance. Microbiol Mol Biol Rev 74(3): 417-433.

52. Hinrichs W, Kisker C, Duvel M, Muller A, Tovar K, Hillen W \& Saenger W. (1994). Structure of the Tet repressor-tetracycline complex and regulation of antibiotic resistance. Sci 264(5157): 418-420.

53. Bouanchaud DH, Scavizzi MR \& Chabbert YA (1968). Elimination by ethidium bromide of antibiotic resistance in Enterobacteria and Staphylococci. Microbiol 54(3): 417-425.

54. Sharma D, Misba L \& Khan AU (2019). Antibiotics versus biofilm: an emerging battleground in microbial communities. Antimicrob Resist Infect Control 8: 76.

55. De la Fuente-Núnnez C, Reffuveille F, Fernandez L \& Rew H (2013). Bacterial biofilm development as a multicellular adaptation: antibiotic resistance and new therapeutic strategies. Curr Opi Microbiol 16: 580-589.

56. Brooun A, Liu S \& Lewis K (2000). A dose-response study of antibiotic resistance in Pseudomonas aeruginosa biofilms. Antimicrob Agents Chemother 44(3): 640646.

57. Mulcahy H, Charron-Mazenod L \& Lewenza S (2008). Extracellular DNA chelates cations and induces antibiotic resistance in Pseudomonas aeruginosa biofilms. PLoS Pathogens 4(11): e1000213. 